\title{
Effect of preoperative antiplatelet or anticoagulation therapy on hemorrhagic complications in patients with traumatic brain injury undergoing craniotomy or craniectomy
}

\author{
Ladina Greuter, MD, ${ }^{1}$ Muriel UIImann, MD, ${ }^{2}$ Luigi Mariani, MD, ${ }^{1,2}$ Raphael Guzman, MD, ${ }^{1,2}$ and \\ Jehuda Soleman, MD ${ }^{1,2}$ \\ ${ }^{1}$ Department of Neurosurgery, University Hospital Basel, and ${ }^{2}$ Faculty of Medicine, University of Basel, Switzerland
}

\begin{abstract}
OBJECTIVE Traumatic brain injury (TBI) is common among the elderly, often treated with antiplatelet (AP) or anticoagulation (AC) therapy, creating new challenges in neurosurgery. In contrast to elective craniotomy, in which AP/AC therapy is mostly discontinued, in TBI usually no delay in treatment can be afforded. The aim of this study was to analyze the effect of AP/AC therapy on postoperative bleeding after craniotomy/craniectomy in TBI.
\end{abstract}

METHODS Postoperative bleeding rates in patients treated with AP/AC therapy (blood thinner group) and in those without AP/AC therapy (control group) were retrospectively compared. Furthermore, univariate and multivariate analyses were conducted to identify risk factors for postoperative bleeding. Lastly, a proportional Cox regression analysis comparing postoperative bleeding events within 14 days in both groups was performed.

RESULTS Of 143 consecutive patients undergoing craniotomy/craniectomy for TBI between 2012 and 2017, 47 (32.9\%) were under AP/AC treatment. No significant difference for bleeding events was observed in univariate $(40.4 \%$ blood thinner group vs $36.5 \%$ control group; $p=0.71$ ) or Cox proportional regression analysis $\left(\log\right.$ rank $\left.\chi^{2}=0.29, p=0.59\right)$. Patients with postoperative bleeding showed a significantly higher mortality rate $(p=0.035)$. In the univariate analysis, hemispheric lesion, acute subdural hematoma, hematological disease, greater extent of midline shift, and pupillary difference were significantly associated with a higher risk of postoperative bleeding. However, in the multivariate regression analysis none of these factors showed a significant association with postoperative bleeding.

CONCLUSIONS Patients treated with AP/AC therapy undergoing craniotomy/craniectomy due to TBI do not appear to have increased rates of postoperative bleeding. Once postoperative bleeding occurs, mortality rates rise significantly. https://thejns.org/doi/abs/10.3171/2019.8.FOCUS19546

KEYWORDS traumatic brain injury; antiplatelet therapy; anticoagulation; hemorrhagic complications

$\mathrm{T}$ RAUMATIC brain injury (TBI) is one of the leading causes of death and disability worldwide. Its incidence in Europe is estimated at 235 per 100,000 people every year, of which $9 \%$ are severe TBI. ${ }^{29}$ Two specific age groups, namely young adults and elderly patients, are most commonly affected. ${ }^{1}$ Many of the latter are treated with antiplatelet (AP) or anticoagulation (AC) therapy for the prevention of cardiovascular and thromboembolic events. However, these patients are simultaneously at risk for hemorrhagic complications, especially following TBI. ${ }^{4,70}$ Due to the increasing age of our population, the incidence of patients suffering from TBI under
AP or AC treatment is increasing, creating a new challenge in neurosurgery. In elective intracranial surgery, $\mathrm{AP}$ or $\mathrm{AC}$ treatment is usually discontinued several days before surgery. ${ }^{19}$ However, in the acute setting, such as in TBI, preoperative discontinuation is not possible and intracranial surgeries are often performed under the effects of AP and AC therapy. Currently, various agents for the reversal of AC treatment are available, while the effect of AP treatment can only be reversed with discontinuation of treatment for at least 3 days. ${ }^{8,13}$ Most large randomized studies on AC or AP therapy in the perioperative phase exclude neurosurgical patients. ${ }^{7}$ Studies on the management

ABBREVIATIONS $\mathrm{AC}=$ anticoagulation; $\mathrm{AP}=$ antiplatelet; $\mathrm{ASA}=$ acetylsalicylic acid; $\mathrm{aSDH}=$ acute subdural hematoma; $\mathrm{CAD}=\mathrm{coronary}$ artery disease; $\mathrm{cSDH}=$ chronic subdural hematoma; $\mathrm{CVI}=$ cerebrovascular insult; $\mathrm{DOAC}=$ direct-acting oral anticoagulant; GCS = Glasgow Coma Scale; INR = international normalized ratio; mRS = modified Rankin Scale; $\mathrm{TBI}$ = traumatic brain injury.

SUBMITTED July 1, 2019. ACCEPTED August 16, 2019.

INCLUDE WHEN CITING DOI: 10.3171/2019.8.FOCUS19546. 
of neurosurgical patients treated with AP or AC therapy undergoing craniotomy, especially in the setting of TBI, are sparse. ${ }^{6,11,20,22,25,27}$ The aim of this study was to analyze hemorrhagic complications in patients treated with AP or AC therapy undergoing craniotomy due to TBI.

\section{Methods}

\section{Study Population and Data Collection}

We retrospectively included consecutive adult patients ( $\geq 18$ years old) undergoing emergency craniotomy or craniectomy for TBI between 2012 and 2017. Data were extracted from our surgical logbook and the patients' medical files. Data on preoperative Glasgow Coma Scale (GCS) score, type of surgery, type of TBI, location of the initial traumatic bleed, hematoma volume, intake of AP/ $\mathrm{AC}$ treatment, discontinuation and resumption time of $\mathrm{AP} / \mathrm{AC}$ treatment, reversal of $\mathrm{AP} / \mathrm{AC}$ treatment, intraoperative blood loss, duration of surgery, pre- and postoperative hemoglobin, international normalized ratio (INR) and platelet count, occurrence of postoperative thromboembolic events and hemorrhagic complications, mortality, clinical outcome (defined as "better," "same," or "worse"), and modified Rankin Scale (mRS) score at last follow-up were collected. Postoperative intracranial bleeding was defined as an abnormal amount of acute intracranial blood on postoperative CT, based on the authors' assessment and the neuroradiologist's report. In addition, we differentiated between overall postoperative intracranial bleeding rate, symptomatic postoperative intracranial bleeding, and intracranial bleeding indicating revision surgery. The study protocol was approved by the local ethics committee (EKNZ in Basel, Switzerland), where patient consent was waived.

\section{Statistical Analysis}

Statistical analysis was conducted using SPSS software (version 21, IBM Corp.). In the main analysis we compared postoperative intracranial bleeding rates and thromboembolic complications between patients treated with AP/AC and those without. In addition, the time to bleed within 14 days after surgery was calculated for each group using a Cox regression analysis, and comparing the groups using a log-rank test. Furthermore, we calculated risk factors for postoperative intracranial bleeding in univariate and multivariate logistic regression analysis. Lastly, we evaluated risk factors for postoperative intracranial bleeding within the different AC/AP types of medication (acetylsalicylic acid [ASA], clopidogrel, vitamin K antagonists, or directacting oral anticoagulants [DOACs]).

Univariate analysis was performed using the Fisher exact test or chi-square test for categorical data and the Mann-Whitney U-test for continuous data. A p value < 0.05 was considered significant.

\section{Results}

\section{Baseline Characteristics}

We included 143 consecutive patients undergoing craniotomy for TBI between the years 2012 and 2017 at our institution. The blood thinner group included 47 patients
(32.8\%) who were under AP or AC treatment and the control group consisted of 96 patients $(67.1 \%)$ without AP or $\mathrm{AC}$ treatment. Patients in the blood thinner group were of significantly higher mean age, suffered significantly more often from acute subdural hematoma (aSDH), showed significantly larger hematoma volumes and larger extent of midline shift on preoperative imaging, significantly more often hemispheric lesions, and suffered more often from cardiovascular disease compared with patients from the control group (Table 1). The groups showed no difference in preoperative GCS score, but preoperative motor symptoms and epilepsy occurred more often in the blood thinner group. Finally, mean intraoperative blood loss was significantly higher in the control group compared to the blood thinner group. The baseline characteristics of both groups are presented in Table 1.

\section{Hemorrhagic Complications, Outcome, and Mortality}

The overall rate of hemorrhagic complications was $37.8 \%(\mathrm{n}=54)$. Out of these, 28 patients $(51.6 \%)$ underwent revision surgery. Baseline characteristics of patients suffering from a hemorrhagic complication compared to patients without are shown in Table 2. Postoperative bleeding rates in the blood thinner group were higher $(\mathrm{n}=$ $19,40.4 \%)$ compared to the control group $(n=28,36.5 \%)$, without showing a significant difference $(\mathrm{p}=0.71)$. Table 3 outlines the distribution of the postoperative bleeding time within the cohort. Of all bleeding events, $79.6 \%$ occurred within the first 3 postoperative days, as opposed to $7.4 \%$ of the bleedings occurring between day 3 and day 14 after surgery, and $13.0 \%$ occurring after the 14th postoperative day. The timing of postoperative bleeding was similar as well, when comparing the bleeding group to the control group (Table 3). Time to bleed was comparable in both groups with a mean time of $9.5 \pm 5.9$ days in the blood thinner group and $10.4 \pm 5.7$ days in the control group (log rank $\chi^{2}=0.29, p=0.59$; Fig. 1). In addition, no significant difference in time to bleed among the different types of $\mathrm{AP} / \mathrm{AC}$ was observed (log rank $\chi^{2}=3.4, \mathrm{p}=0.49$; Fig. 2). Thromboembolic complications occurred in 15 patients $(10.5 \%)$ in the cohort, without any significant differences between the groups (Fig. 3). Overall mortality rate was $16.9 \%$ while no significant difference was noted between patients in the blood thinner group (19\%) and the control group (14.7\%). Similarly, postoperative GCS score, postoperative clinical presentation (better, same, worse), and mRS score did not differ significantly between the groups.

\section{Risk Factors for Postoperative Hemorrhage}

Univariate analysis showed a significant association between hemispheric lesion at initial diagnosis, aSDH at presentation, hematological disease, greater extent of midline shift in preoperative images, and pupillary difference with a higher rate of postoperative bleeding (Table 2). AP or $\mathrm{AC}$ treatment was not found to be a risk factor for postoperative bleeding. Multivariate logistic regression analysis failed, however, to single out one of the parameters above as a potential risk factor for postoperative hemorrhagic complications. Total discontinuation time of AP/AC did not appear to affect bleeding rates (Table 4; $\mathrm{p}=0.52$ ), 
TABLE 1. Baseline characteristics of the control group and the blood thinner group

\begin{tabular}{|c|c|c|c|}
\hline Variable & $\begin{array}{l}\text { Control } \\
(n=96)\end{array}$ & $\begin{array}{l}\text { Blood Thinner } \\
\quad(n=47)\end{array}$ & $\begin{array}{c}\mathrm{p} \\
\text { Value }\end{array}$ \\
\hline Males & $67(69.8)$ & $30(63.8)$ & NS \\
\hline Mean age $\pm S D$, yrs & $53.6 \pm 19.9$ & $74.7 \pm 10.3$ & $<0.001$ \\
\hline Type of hematoma & & & 0.008 \\
\hline aSDH & $33(34.4)$ & $29(61.7)$ & \\
\hline $\mathrm{cSDH}$ & $6(6.3)$ & $3(6.4)$ & \\
\hline $\mathrm{aEDH}$ & $33(34.4)$ & $3(6.4)$ & \\
\hline $\mathrm{ICH}$ & $5(5.2)$ & $3(6.4)$ & \\
\hline Combined & $18(18.8)$ & $8(17)$ & \\
\hline Other & $1(1)$ & $1(2.1)$ & \\
\hline Type of surgery & & & $<0.001$ \\
\hline Craniotomy & $53(55.2)$ & $32(68.1)$ & \\
\hline Minicraniotomy & $3(3.1)$ & $4(8.5)$ & \\
\hline Craniectomy & $40(41.7)$ & $11(23.4)$ & \\
\hline Preop GCS score & & & NS \\
\hline $3-8$ & $52(54.2)$ & $22(46.8)$ & \\
\hline $9-13$ & $21(21.9)$ & $13(27.7)$ & \\
\hline $14-15$ & $23(24)$ & $12(25.5)$ & \\
\hline Region of hematoma & & & 0.002 \\
\hline Hemispheric & $53(55.2)$ & $43(91.5)$ & \\
\hline Frontal & $9(9.4)$ & $1(2.2)$ & \\
\hline Parietal & $6(6.3)$ & $0(0)$ & \\
\hline Temporal & $11(11.5)$ & $1(2.1)$ & \\
\hline Occipital & $1(1)$ & $0(0)$ & \\
\hline Skull base & $16(16.7)$ & $2(4.3)$ & \\
\hline $\begin{array}{l}\text { Mean hematoma vol } \pm \mathrm{SD} \text {, } \\
\mathrm{ml}\end{array}$ & $357.2 \pm 390$ & $418.1 \pm 259.3$ & 0.015 \\
\hline Mean midline shift $\pm \mathrm{SD}, \mathrm{mm}$ & $7.1 \pm 5.3$ & $10.6 \pm 9$ & 0.014 \\
\hline Pupillary abnormality & $34(35.4)$ & $13(27.7)$ & NS \\
\hline Preop motor deficits & $18(18.8)$ & $22(46.8)$ & $<0.001$ \\
\hline Preop sensory deficits & $5(5.2)$ & $7(14.9)$ & NS \\
\hline Preop epilepsy & $4(4.2)$ & $7(14.9)$ & 0.04 \\
\hline Preop coma & $55(57.3)$ & $20(42.6)$ & NS \\
\hline Preop cranial nerve palsy & $4(4.2)$ & $6(12.8)$ & NS \\
\hline $\begin{array}{l}\text { Mean preop hemoglobin } \pm \\
\text { SD, g/L }\end{array}$ & $120.7 \pm 22$ & $119.1 \pm 110.3$ & NS \\
\hline $\begin{array}{l}\text { Mean postop hemoglobin } \pm \\
\text { SD, g/L }\end{array}$ & $103 \pm 18.2$ & $103.4 \pm 16.4$ & NS \\
\hline $\begin{array}{l}\text { Mean preop platelets } \pm S D \text {, } \\
\mathrm{g} / \mathrm{L}\end{array}$ & $211.3 \pm 22.1$ & $229.2 \pm 110.3$ & NS \\
\hline $\begin{array}{l}\text { Mean postop platelets } \pm \\
\text { SD, g/L }\end{array}$ & $189 \pm 89.5$ & $203.7 \pm 100.6$ & NS \\
\hline Mean preop INR \pm SD & $1.1 \pm 0.2$ & $1.4 \pm 0.7$ & NS \\
\hline Mean postop INR \pm SD & $1.1 \pm 0.3$ & $1 \pm 0.1$ & NS \\
\hline Hypertension & $15(15.6)$ & $36(76.6)$ & $<0.001$ \\
\hline CAD & $0(0)$ & $20(42.6)$ & $<0.001$ \\
\hline Diabetes mellitus & $1(1)$ & $15(31.9)$ & $<0.001$ \\
\hline $\mathrm{CVI}$ & $1(1)$ & $11(23.4)$ & $<0.001$ \\
\hline Atrial fibrillation & $4(4.2)$ & $15(31.9)$ & $<0.001$ \\
\hline
\end{tabular}

CONTINUED IN NEXT COLUMN »
» CONTINUED FROM PREVIOUS COLUMN

TABLE 1. Baseline characteristics of the control group and the blood thinner group

\begin{tabular}{lccc}
\hline \multicolumn{1}{c}{ Variable } & $\begin{array}{c}\text { Control } \\
(\mathrm{n}=96)\end{array}$ & $\begin{array}{c}\text { Blood Thinner } \\
(\mathrm{n}=47)\end{array}$ & $\begin{array}{c}\mathrm{p} \\
\text { Value }\end{array}$ \\
\hline Hematological disease & $6(6.3)$ & $4(8.5)$ & NS \\
\hline $\begin{array}{l}\text { Mean hospital time } \pm \mathrm{SD}, \\
\text { days }\end{array}$ & $13.6 \pm 9.8$ & $13.9 \pm 9$ & $\mathrm{NS}$ \\
\hline
\end{tabular}

aEDH = acute epidural hematoma; $\mathrm{ICH}$ = intracerebral hematoma; $\mathrm{NS}=$ nonsignificant.

All values presented as number (\%) of patients or mean $\pm S D$, if not otherwise specified. Boldface type indicates statistical significance.

however, in the majority of patients AP/AC was discontinued for more than 14 days. Preoperative discontinuation time was more equally distributed, in which $63.8 \%$ of the patients' AP/AC treatment was not discontinued preoperatively $(12.8 \%, 1-3$ days; $17.0 \%, 4-5$ days; $2.1 \%, 6-7$ days; and $4.3 \%, 7-14$ days), but this did not appear to affect bleeding rates either $(p=0.325)$. Patients with postoperative bleeding showed significantly higher thromboembolic event rates (18.5\%) and mortality rates (25.9\%) compared to patients without postoperative bleeding (5.6\% and $11.2 \%$ respectively; $\mathrm{p}=0.017$ and $\mathrm{p}=0.035$, respectively).

\section{Discussion}

Based on our data, AP/AC treatment does not appear to affect postoperative bleeding complications in patients undergoing craniotomy due to TBI. aSDH, hemispheric pathology, greater extent of midline shift on preoperative imaging, type of surgery, evidence of a hematological disease, and pupillary abnormality were significant risk factors for postoperative bleeding in univariate analysis. However, in the multivariate logistic regression analysis none of those factors showed a significant association with postoperative bleeding. Thromboembolic event rate and mortality rate were significantly higher in patients suffering from postoperative bleeding, while rates of good outcome in survivors did not appear to be affected by postoperative bleeding. The vast majority (79.6\%) of postoperative bleedings occurred within 96 hours after surgery, while the time to bleeding within 14 days did not differ significantly between the groups. In addition, time to bleeding within 14 days showed no significant difference between the different types of AP/AC treatment. ${ }^{28}$

\section{Management of APIAC Treatment in Neurosurgical Patients}

To date, the literature on AP/AC therapy in neurosurgery in general and specifically in TBI is sparse. A study by Han et al. showed a postoperative bleeding rate of $52.5 \%$ after decompressive craniectomy for TBI in patients treated with AP therapy. ${ }^{11}$ The rate described is higher than in our cohort, however they only included patients undergoing hemicraniectomy with a midline shift $>5 \mathrm{~mm}$. AP treatment was shown to be associated with higher mortality rates in TBI. ${ }^{22,25,31}$ However, these studies 
TABLE 2. Baseline characteristics of patients with and without postoperative hemorrhagic complications

\begin{tabular}{|c|c|c|c|}
\hline Variable & $\begin{array}{l}\text { Bleeding } \\
(n=54)\end{array}$ & $\begin{array}{l}\text { No Bleeding } \\
\quad(n=89)\end{array}$ & $\begin{array}{c}p \\
\text { Value }\end{array}$ \\
\hline Males & $36(66.7)$ & $61(68.5)$ & NS \\
\hline Mean age $\pm S D$, yrs & $74.7 \pm 10.3$ & $53.6 \pm 20$ & $<0.001$ \\
\hline Type of hematoma & & & 0.045 \\
\hline aSDH & $32(59.3)$ & $30(33.7)$ & \\
\hline $\mathrm{cSDH}$ & $4(7.4)$ & $5(5.6)$ & \\
\hline $\mathrm{aEDH}$ & $9(16.7)$ & $27(30.3)$ & \\
\hline $\mathrm{ICH}$ & $1(1.9)$ & $7(7.9)$ & \\
\hline Combined & $7(13)$ & $19(21.3)$ & \\
\hline Other & $1(1.9)$ & $1(1.1)$ & \\
\hline Type of surgery & & & 0.038 \\
\hline Craniotomy & $26(48.1)$ & $59(66.3)$ & \\
\hline Minicraniotomy & $2(3.8)$ & $5(5.6)$ & \\
\hline Craniectomy & $26(48.1)$ & $25(28.1)$ & \\
\hline Preop GCS score & & & NS \\
\hline $3-8$ & $26(48.1)$ & $59(66.3)$ & \\
\hline $9-13$ & $2(3.7)$ & $5(5.6)$ & \\
\hline $14-15$ & $26(48.2)$ & $25(28.1)$ & \\
\hline Region of hematoma & & & 0.022 \\
\hline Hemispheric & $44(81.5)$ & $52(58.4)$ & \\
\hline Frontal & $0(0)$ & $10(11.2)$ & \\
\hline Parietal & $2(3.7)$ & $4(4.5)$ & \\
\hline Temporal & $3(5.6)$ & $9(10.1)$ & \\
\hline Occipital & $1(1.9)$ & $0(0)$ & \\
\hline Skull base & $4(7.4)$ & $14(15.7)$ & \\
\hline $\begin{array}{l}\text { Mean hematoma vol } \pm \\
\quad \text { SD, ml }\end{array}$ & $366.9 \pm 239.4$ & $383.1 \pm 405.3$ & NS \\
\hline $\begin{array}{l}\text { Mean midline shift } \pm \text { SD, } \\
\quad \mathrm{mm}\end{array}$ & $9.5 \pm 5.6$ & $7.6 \pm 7.5$ & 0.018 \\
\hline AP/AC & $19(35.2)$ & $28(31.5)$ & NS \\
\hline Type of AP/AC & & & NS \\
\hline None & $35(64.8)$ & $61(68.5)$ & \\
\hline ASA & 8 (14.8) & $12(13.5)$ & \\
\hline Clopidogrel & $2(3.7)$ & $1(1.1)$ & \\
\hline Vitamin $\mathrm{K}$ antagonist & $3(5.6)$ & $9(10.1)$ & \\
\hline DOAC & $4(7.4)$ & $2(2.2)$ & \\
\hline Multiple & $2(3.7)$ & $4(4.5)$ & \\
\hline Pupillary abnormality & $23(42.6)$ & $24(27)$ & 0.041 \\
\hline Preop motor deficits & $18(33.3)$ & $22(24.7)$ & NS \\
\hline Preop sensory deficits & $6(11.1)$ & $6(6.7)$ & NS \\
\hline Preop epilepsy & $6(11.1)$ & $5(5.6)$ & NS \\
\hline Preop coma & $30(55.6)$ & $45(50.6)$ & NS \\
\hline Preop cranial nerve palsy & $6(11.1)$ & $4(4.5)$ & NS \\
\hline $\begin{array}{l}\text { Mean preop hemoglobin } \\
\quad \pm S D, g / L\end{array}$ & $119.1 \pm 23.3$ & $120.7 \pm 22.1$ & NS \\
\hline $\begin{array}{l}\text { Mean preop platelets } \pm \\
\text { SD, g/L }\end{array}$ & $229.2 \pm 110.3$ & $211.3 \pm 92$ & NS \\
\hline Mean preop INR \pm SD & $1.4 \pm 0.8$ & $1.1 \pm 0.2$ & NS \\
\hline
\end{tabular}

» CONTINUED FROM PREVIOUS COLUMN

TABLE 2. Baseline characteristics of patients with and without postoperative hemorrhagic complications

\begin{tabular}{lccc}
\hline \multicolumn{1}{c}{ Variable } & $\begin{array}{c}\text { Bleeding } \\
(\mathrm{n}=54)\end{array}$ & $\begin{array}{c}\text { No Bleeding } \\
(\mathrm{n}=89)\end{array}$ & $\begin{array}{c}\mathrm{p} \\
\text { Value }\end{array}$ \\
\hline Hypertension & $18(33.3)$ & $33(37.1)$ & $\mathrm{NS}$ \\
\hline $\mathrm{CAD}$ & $7(13)$ & $13(14.6)$ & $\mathrm{NS}$ \\
\hline Diabetes mellitus & $3(5.6)$ & $13(14.6)$ & $\mathrm{NS}$ \\
\hline $\mathrm{CVI}$ & $5(9.3)$ & $7(7.9)$ & $\mathrm{NS}$ \\
\hline Atrial fibrillation & $6(11.1)$ & $13(14.6)$ & $\mathrm{NS}$ \\
\hline Hematological disease & $7(13)$ & $3(3.4)$ & $\mathbf{0 . 0 4 2}$ \\
\hline $\begin{array}{l}\text { Thromboembolic compli- } \\
\text { cations }\end{array}$ & $10(18.5)$ & $5(5.6)$ & $\mathbf{0 . 0 1 7}$ \\
\hline $\begin{array}{l}\text { Mean hospital time } \pm \mathrm{SD}, \\
\text { days }\end{array}$ & $13.4 \pm 8.6$ & $13.9 \pm 9.9$ & $\mathrm{NS}$ \\
\hline
\end{tabular}

All values presented as number $(\%)$ of patients or mean $\pm S D$, if not otherwise specified. Boldface type indicates statistical significance.

also included patients who were treated conservatively for TBI, and therefore no adequate comparison to our cohort can be made. Palmer et al. analyzed all patients undergoing craniotomy at their institution over a period of 5 years and identified AP therapy as the most common associated risk factor for postoperative bleeding. Other identified risk factors were emergency surgery for TBI, aneurysms, and meningioma resection. ${ }^{26}$ Nittby et al. retrospectively analyzed all their neurosurgical operations over 3 years, resulting in 7055 surgeries, and identified AP/AC treatment as a risk factor for early postoperative deterioration. ${ }^{23}$

However, in elective brain tumor surgery several studies showed no effect of ASA on postoperative bleeding and clinical outcome. Recently, Hanalioglu et al. conducted a study showing no effect of ASA on hemorrhagic complications in elective craniotomies for brain tumors. In addition, ASA was considered beneficial in patients with a high risk of thromboembolic complications. ${ }^{12}$ Two other studies confirmed these findings and consider brain tumor surgery safe with continuation of ASA treatment during the perioperative phase. ${ }^{24,27}$

Early ASA resumption or even continued perioperative intake was shown to be safe for burr-hole drainage in chronic subdural hematoma (cSDH) as well as shunt placement. ${ }^{9,10,14,15,17}$ To our knowledge, there are no randomized

TABLE 3. Distribution of bleeding time within the cohort

\begin{tabular}{lccc}
\hline Time After Op & Total Cohort & AP/AC Cohort & No AP/AC Cohort \\
\hline$<24$ hrs & $9(16.7)$ & $3(15.8)$ & $6(17.1)$ \\
\hline $24-48$ hrs & $24(44.4)$ & $8(42.1)$ & $16(45.7)$ \\
\hline $48-96$ hrs & $10(18.5)$ & $5(26.3)$ & $5(14.3)$ \\
\hline $3-14$ days & $4(7.4)$ & $2(10.5)$ & $2(5.7)$ \\
\hline$>14$ days & $7(13.0)$ & $1(5.3)$ & $6(17.1)$ \\
\hline Total & $54(100)$ & $19(100)$ & $35(100)$ \\
\hline
\end{tabular}

Note that general bleedings occurring after 14 days were not related to the initial trauma. All values represent number (\%) of patients. 


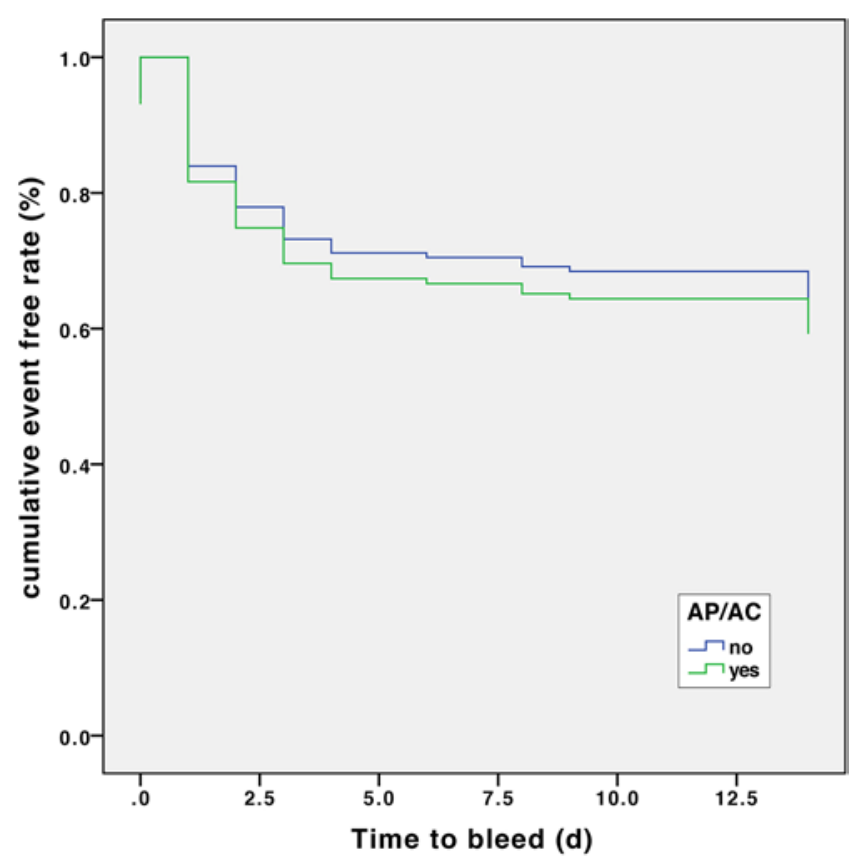

FIG. 1. Cox regression analysis comparing postoperative bleeding in the control (no AP/AC) and blood thinner (yes AP/AC) groups.

controlled trials (RCTs) analyzing bleeding risk with continued perioperative AP/AC treatment in neurosurgery. A protocol for an ongoing RCT of continued ASA treatment for patients undergoing surgical evacuation of cSDH has been published and the study is currently ongoing. ${ }^{16}$

The use of direct oral anticoagulants (DOACs) has become more frequent, creating a further challenge in the perioperative management of neurosurgical patients. A study by Croci et al. showed that a short preoperative discontinuation period might increase bleeding rates after craniotomy. ${ }^{5}$

At our institution, the perioperative management of patients with TBI in need of urgent craniotomy/craniectomy is to reverse the effect of AP/AC medication, when possible. AP medication is not actively reversed, but if the surgeon feels intraoperative bleeding is increased, tranexamic acid or platelet transfusions will be applied. For patients treated with $\mathrm{AC}$ we normalize the INR to a value above 1.2 using vitamin $\mathrm{K}$, fresh frozen plasma, and/ or coagulation factors concentrate (Beriplex). For the first 3 days after surgery, AP/AC treatment is generally discontinued, while in patients with a history of thromboembolic events (such as myocardial infarction or pulmonary embolism), and no rebleeding on postoperative imaging, AP treatment will be continued early (after 2-3 days), while for AC therapy patients, we will start the patient on intravenous unfractionated heparin and once the patient is clinically stable for a few days, restart the patient on oral AC medication. In all other patients, AP/AC treatment is discontinued for at least 14 days after surgery. Postoperative management is decided on a case-by-case basis, often after a thorough discussion within the neurosurgical team and after consulting the cardiologists, hematologists, and intensive care physicians if needed. International guide-

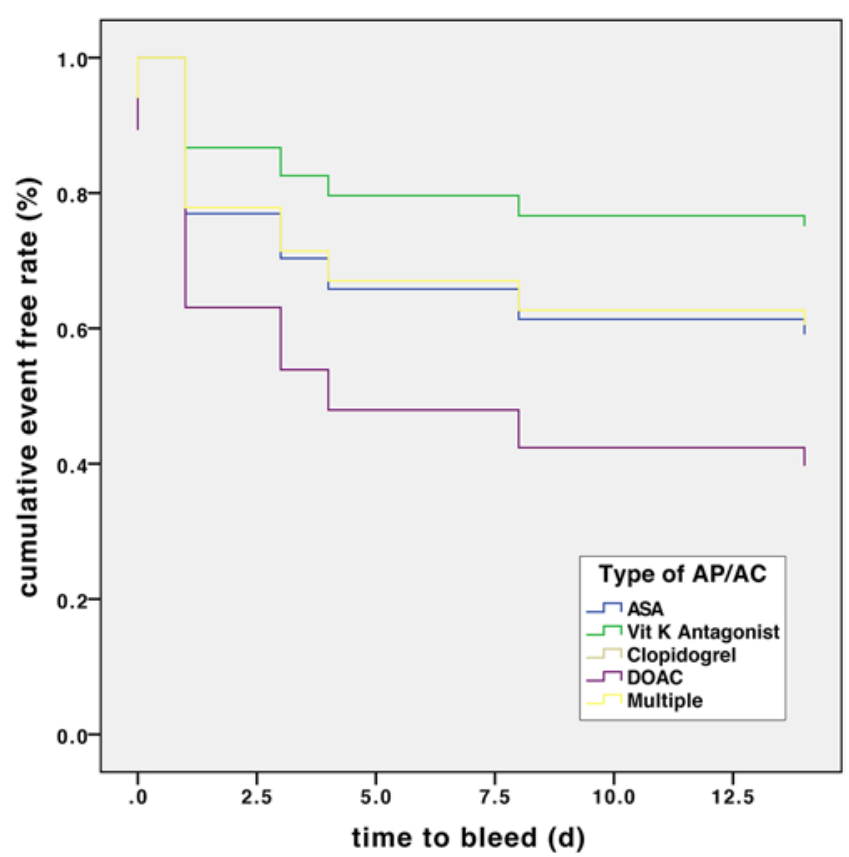

FIG. 2. Cox regression analysis comparing the different types of AP/AC agents in terms of postoperative bleeding rate. There were no significant differences among the different medication types.

lines for the management of patients with TBI undergoing craniotomy/craniectomy continues to be lacking due to the paucity of well-designed studies, therefore the decision-making in these patients remains an ongoing debate and a case-by-case decision.

Clearly further studies focusing on AP/AC therapy in general, and specifically on the different subgroups of blood thinners, as well as the different neurosurgical pathologies are necessary. Especially in patients suffering from TBI, in which the preoperative discontinuation of $\mathrm{AP} / \mathrm{AC}$ treatment is not possible and the risk for thromboembolic events (as well as bleeding events) is markedly elevated, further large prospective studies and management guidelines are needed.

\section{Risk of Thromboembolic Complications}

As expected, patients in the blood thinner group had significantly higher rates of coronary artery disease (CAD), hypertension, cerebrovascular insult (CVI) and diabetes mellitus compared to the control group, but there were no significant differences in thromboembolic events. Similar cardiovascular risk profiles were observed for patients with AP treatment in two retrospective cohort studies for elective craniotomies. In both studies thromboembolic complications did not differ among different groups. ${ }^{12,27}$ This might be due to the fact that the intake of AP/AC medication up to the surgery protected the patients, with a high risk for thrombotic complication. The POISE-2 study showed that in patients with primary ASA prophylaxis undergoing noncardiac surgery, hemorrhagic complications were increased, with no significant effect on the prevention of myocardial infarction. ${ }^{7}$ However, in a different study, ASA treatment as a secondary prevention 


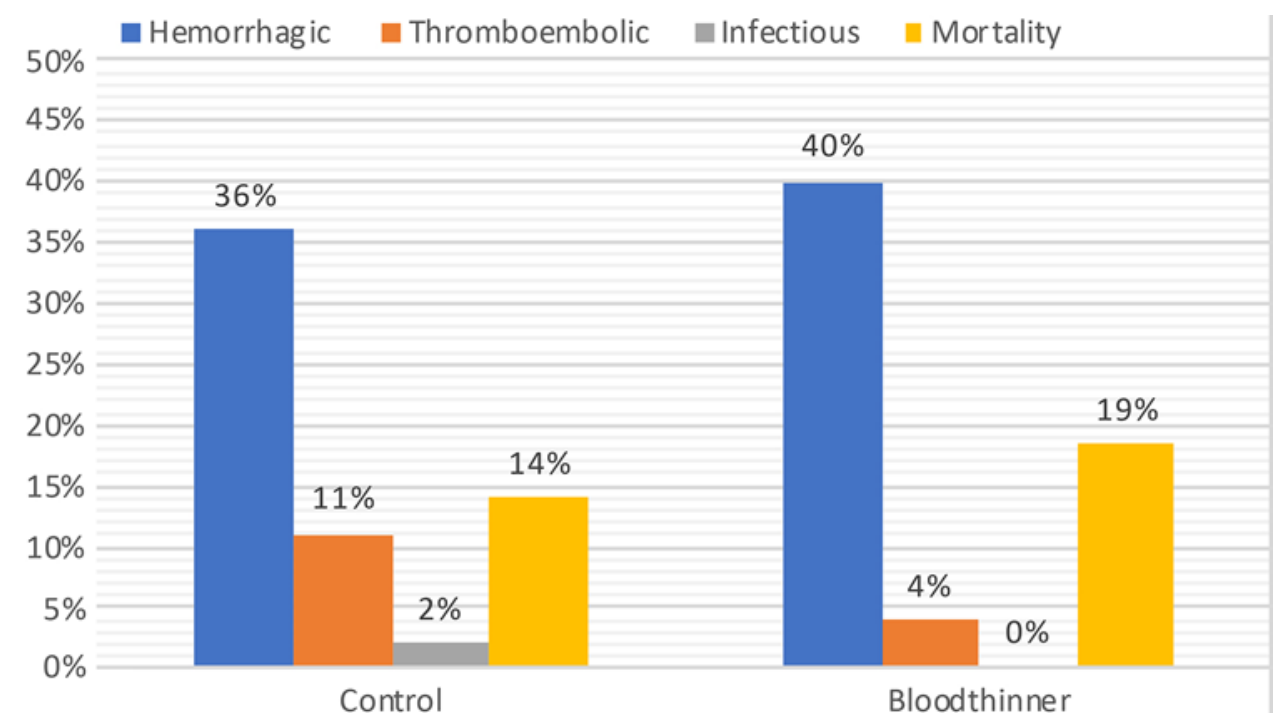

FIG. 3. Complication rates in the blood thinner and control groups.

has been proven beneficial in preventing cardiovascular complications. ${ }^{2}$ These studies excluded neurosurgical patients, and therefore extrapolation of their results to neurosurgical patients is difficult.

At our institution, in the vast majority of the cases lowmolecular-weight heparin is administered 24-48 hours after surgery for postoperative thrombosis prophylaxis. Exceptions are patients with large acute hematomas, or patients with postoperative bleedings. This might explain our findings, that patients suffering a postoperative bleed had a higher rate of thromboembolic events. Based on the available literature it seems that early prophylactic heparin administration appears to be safe after neurosurgical procedures, specifically in TBI., ${ }^{3,18,21}$ Regardless, the management of thrombosis prophylaxis in patients with postoperative bleeding after craniotomy/craniectomy in TBI remains difficult due to a paucity of evidence.

\section{Mortality Rates in TBI Patients Treated With APIAC}

Patients with AP/AC treatment were not shown to have significantly higher mortality rates in TBI. ${ }^{6}$ Furthermore, no difference in 7-day mortality rates with respect to AP intake was noted for patients undergoing decompressive hemicraniectomy. ${ }^{11}$ This data is consistent with our results, which indicates that although patients suffering postoper-

TABLE 4. Distribution of total discontinuation time of APIAC agents within the blood thinner group

\begin{tabular}{crrr}
\hline Discontinuation Time (days) & No. of Patients & Bleeding & No Bleeding \\
\hline$<7$ & $2(4.3)$ & $1(5.3)$ & $1(3.6)$ \\
\hline $7-14$ & $5(10.6)$ & $1(5.3)$ & $4(14.3)$ \\
\hline $15-30$ & $32(68.1)$ & $14(73.7)$ & $18(64.3)$ \\
\hline$>30$ & $8(17.0)$ & $3(15.8)$ & $5(17.9)$ \\
\hline Total & $47(100)$ & $19(100)$ & $28(100)$ \\
\hline
\end{tabular}

All values represent number (\%) of patients. ative bleeding have higher mortality rates, postoperative bleeding does not appear to be directly affected by the preoperative intake of AP/AC medications.

\section{Limitations}

This retrospective study is subject to all the limitations of data collection inherent in such study designs. The intraoperative management of patients under AP/AC treatment was based on the treating physician's judgment. The discontinuation time and resumption time of AP/AC therapy after surgery was not uniform for all patients. Most patients discontinued AP/AC treatment for more than 14 days and therefore we cannot make recommendations on the perioperative management of $\mathrm{AP} / \mathrm{AC}$ treatment based on our data. Perioperative discontinuation of AP/AC therapy was distributed more evenly, with many patients continuing treatment up until the surgery. Therefore, our results showing no effect on postoperative bleeding rate are more firm and valid. Furthermore, we did not analyze any other concomitant injuries apart from TBI, which might have influenced perioperative AP/AC management, as well as the outcome and mortality rates of our cohort. Lastly, ASA or P2Y12 response assays as well as thromboelastography testing was not routinely performed in our cohort. Therefore, we could not assess the true effect of the AP/AC treatment and its possible influence on bleeding rates.

\section{Conclusions}

Preoperative AP/AC intake does not appear to influence the rate of postoperative bleeding, outcome, or mortality in patients undergoing craniotomy or craniectomy due to TBI. Most postoperative bleedings occur within the first 3 days, regardless of whether the patient is treated with $\mathrm{AP} /$ $\mathrm{AC}$ medication prior to surgery. Potential risk factors for postoperative hemorrhagic complications after craniotomy for TBI might be the occurrence of an aSDH, hemispheric pathologies, craniectomy, preoperative pupil abnormality, 
and greater extent of midline shift on preoperative imaging, but further studies are needed to confirm these results. Whether or not to discontinue AP/AC treatment in patients undergoing emergency craniotomy/craniectomy in TBI, and the duration of these agents should remain discontinued, will need to be the focus of future prospective studies.

\section{References}

1. Bruns J Jr, Hauser WA: The epidemiology of traumatic brain injury: a review. Epilepsia 44 (s10):2-10, 2003

2. Baigent C, Blackwell L, Collins R, Emberson J, Godwin J, Peto R, et al: Aspirin in the primary and secondary prevention of vascular disease: collaborative meta-analysis of individual participant data from randomised trials. Lancet 373:1849-1860, 2009

3. Constantini S, Kanner A, Friedman A, Shoshan Y, Israel Z, Ashkenazi E, et al: Safety of perioperative minidose heparin in patients undergoing brain tumor surgery: a prospective, randomized, double-blind study. J Neurosurg 94:918-921, 2001

4. Copland M, Walker ID, Tait RC: Oral anticoagulation and hemorrhagic complications in an elderly population with atrial fibrillation. Arch Intern Med 161:2125-2128, 2001

5. Croci DM, Kamenova M, Guzman R, Mariani L, Soleman J: Novel oral anticoagulants in patients undergoing cranial surgery. World Neurosurg 105:841-848, 2017

6. Cull JD, Sakai LM, Sabir I, Johnson B, Tully A, Nagy K, et al: Outcomes in traumatic brain injury for patients presenting on antiplatelet therapy. Am Surg 81:128-132, 2015

7. Devereaux PJ, Mrkobrada M, Sessler DI, Leslie K, AlonsoCoello P, Kurz A, et al: Aspirin in patients undergoing noncardiac surgery. N Engl J Med 370:1494-1503, 2014

8. Gressenberger P: Reversal strategies in patients treated with direct oral anticoagulants. Vasa 48:389-392, 2019

9. Guha D, Coyne S, Macdonald RL: Timing of the resumption of antithrombotic agents following surgical evacuation of chronic subdural hematomas: a retrospective cohort study. J Neurosurg 124:750-759, 2016

10. Guha D, Macdonald RL: Perioperative management of anticoagulation. Neurosurg Clin N Am 28:287-295, 2017

11. Han H, Koh EJ, Choi H, Kim BC, Yang SY, Cho KT: The effect of preoperative antiplatelet therapy on hemorrhagic complications after decompressive craniectomy in patients with traumatic brain injury. Korean J Neurotrauma 12:61-66, 2016

12. Hanalioglu S, Sahin B, Sahin OS, Kozan A, Ucer M, Cikla U, et al: Effect of perioperative aspirin use on hemorrhagic complications in elective craniotomy for brain tumors: results of a single-center, retrospective cohort study. J Neurosurg [epub ahead of print April 5, 2019. DOI: 10.3171/2018.12.JNS182483]

13. Jimenez AH, Stubbs ME, Tofler GH, Winther K, Williams $\mathrm{GH}$, Muller JE: Rapidity and duration of platelet suppression by enteric-coated aspirin in healthy young men. Am J Cardiol 69:258-262, 1992

14. Kamenova M, Croci D, Guzman R, Mariani L, Soleman $\mathrm{J}$ : Low-dose acetylsalicylic acid and bleeding risks with ventriculoperitoneal shunt placement. Neurosurg Focus 41(3):E4, 2016

15. Kamenova M, Lutz K, Schaedelin S, Fandino J, Mariani L, Soleman J: Does early resumption of low-dose aspirin after evacuation of chronic subdural hematoma with burr-hole drainage lead to higher recurrence rates? Neurosurgery 79:715-721, 2016

16. Kamenova M, Mueller C, Coslovsky M, Guzman R, Mariani $\mathrm{L}$, Soleman J: Low-dose aspirin and burr-hole drainage of chronic subdural hematoma: study protocol for a randomized controlled study. Trials 20:70, 2019

17. Kamenova M, Nevzati E, Lutz K, Dolp A, Fandino J, Mariani $\mathrm{L}$, et al: Burr-hole drainage for chronic subdural hematoma under low-dose acetylsalicylic acid: a comparative risk analysis study. World Neurosurg 100:594-600, 2017

18. Kim J, Gearhart MM, Zurick A, Zuccarello M, James L, Luchette FA: Preliminary report on the safety of heparin for deep venous thrombosis prophylaxis after severe head injury. J Trauma 53:38-43, 2002

19. Korinth MC: Low-dose aspirin before intracranial surgeryresults of a survey among neurosurgeons in Germany. Acta Neurochir (Wien) 148:1189-1196, 2006

20. Lee AT, Gagnidze A, Pan SR, Sookplung P, Nair B, Newman SF, et al: Preoperative low-dose aspirin exposure and outcomes after emergency neurosurgery for traumatic intracranial hemorrhage in elderly patients. Anesth Analg 125:514-520, 2017

21. Licci M, Kamenova M, Guzman R, Mariani L, Soleman J: Influence of postoperative thrombosis prophylaxis on the recurrence of chronic subdural hematoma after burr-hole drainage. Crit Care Med 46:e26-e32, 2018

22. Mina AA, Knipfer JF, Park DY, Bair HA, Howells GA, Bendick PJ: Intracranial complications of preinjury anticoagulation in trauma patients with head injury. J Trauma 53:668-672, 2002

23. Nittby HR, Maltese A, Ståhl N: Early postoperative haematomas in neurosurgery. Acta Neurochir (Wien) 158:837-846, 2016

24. Ogawa Y, Tominaga T: Sellar and parasellar tumor removal without discontinuing antithrombotic therapy. J Neurosurg 123:794-798, 2015

25. Ohm C, Mina A, Howells G, Bair H, Bendick P: Effects of antiplatelet agents on outcomes for elderly patients with traumatic intracranial hemorrhage. J Trauma 58:518-522, 2005

26. Palmer JD, Sparrow OC, Iannotti F: Postoperative hematoma: a 5-year survey and identification of avoidable risk factors. Neurosurgery 35:1061-1065, 1994

27. Rahman M, Donnangelo LL, Neal D, Mogali K, Decker M, Ahmed MM: Effects of perioperative acetyl salicylic acid on clinical outcomes in patients undergoing craniotomy for brain tumor. World Neurosurg 84:41-47, 2015

28. Robba C, Bertuetti R, Rasulo F, Bertuccio A, Matta B: Coagulation management in patients undergoing neurosurgical procedures. Curr Opin Anaesthesiol 30:527-533, 2017

29. Tagliaferri F, Compagnone C, Korsic M, Servadei F, Kraus J: A systematic review of brain injury epidemiology in Europe. Acta Neurochir (Wien) 148:255-268, 2006

30. Williams CD, Chan AT, Elman MR, Kristensen AH, Miser WF, Pignone MP, et al: Aspirin use among adults in the U.S.: results of a national survey. Am J Prev Med 48:501-508, 2015

31. Wong DK, Lurie F, Wong LL: The effects of clopidogrel on elderly traumatic brain injured patients. J Trauma 65:13031308,2008

\section{Disclosures}

The authors report no conflict of interest concerning the materials or methods used in this study or the findings specified in this paper.

\section{Author Contributions}

Conception and design: Greuter, Soleman. Acquisition of data: Greuter, Ullmann. Analysis and interpretation of data: Greuter, Soleman. Drafting the article: Greuter. Critically revising the article: Mariani, Guzman, Soleman. Reviewed submitted version of manuscript: Greuter, Soleman. Statistical analysis: Soleman. Study supervision: Soleman.

\section{Correspondence}

Ladina Greuter: University Hospital Basel, Switzerland. ladinaaurea.greuter@gmail.com. 